\title{
Comparative study of line roughness metrics of chemically amplified and inorganic resists for extreme ultraviolet
}

\author{
Roberto Fallica, [f Elizabeth Buitrago, and Yasin Ekinci \\ Paul Scherrer Institute, 5232 Villigen PSI, Switzerland
}

\begin{abstract}
We present a comprehensive comparative study of the roughness metrics of different resists. Dense line/space of polymethyl methacrylate, hydrogen silsesquioxane, a metal oxide-based resist, and different chemically amplified resists (CARs) have been patterned by extreme ultraviolet interference lithography. All three line width roughness (LWR) metrics: the root-mean-square (r.m.s.) roughness value $\sigma_{\mathrm{LWR}}$, the correlation length $\xi$, and the roughness exponent $\alpha$, were extracted by metrological analysis of top-down SEM images. We found that all metrics are required to fully describe the overall roughness of each resist. Our measurements indicate that in fact, a few of the state-of-the-art resists tested here can meet the International Technology Roadmap for Semiconductors requirements for $\sigma_{\text {LWR }}$. The correlation length $\xi$ was also found to be considerably higher in polymer-based materials in comparison to nonpolymers. Finally, the roughness exponent $\alpha$, interpreted using the concept of fractal geometry, was found to be mainly affected by acid diffusion in CARs, where it produces line edges with a higher complexity than in non-CAR resists. These results indicate that the different resists platforms show very different LWR metrics and roughness is not manifested only in the $\sigma_{\mathrm{LWR}}$ but in all parameters. Therefore, all roughness metrics should be taken into account when comparing the performance among different resists since they ultimately have a substantial impact on device performance. $\odot 2016$ Society of Photo-Optical Instrumentation Engineers (SPIE) [DOI: 10.1117/1.JMM.15.3.034003]
\end{abstract}

Keywords: line roughness; LWR; line width roughness; power spectral density; correlation length; roughness exponent. Paper 16050P received May 4, 2016; accepted for publication Aug. 1, 2016; published online Aug. 18, 2016.

\section{Introduction}

The line width roughness (LWR) metric of the resist image is of crucial technological importance because it directly translates into, i.e., gate length dimension variations-during plasma etch pattern transfer-and ultimately impacts device performance and chip reliability. Early studies have found that the threshold voltage and other electrical characteristics in Fin field effect transistors are affected by the LWR due to fluctuations in the gate length, width, and related geometrical

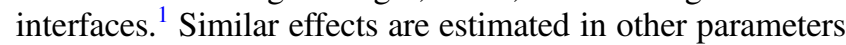
such as cut-off frequency, gate capacitance, off current $\left(I_{\text {off }}\right)$, and so on.

There are several LWR sources such as aerial image quality, mask roughness, photon shot noise, secondary electron blur, acid diffusion, statistics of deprotection and quenching events, molecular size, thickness, development, and so on. Although some of these causes are systematic and others are stochastic, the measured roughness is eventually a stochastic one-dimensional spatial signal. As such, the analysis of roughness in the domain of spatial frequency was introduced and these descriptors were defined: the power spectral density (PSD), the $\sigma$ versus line length curve, and the height-

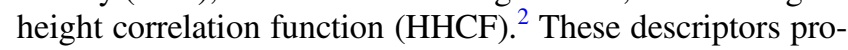
vide three metrics that altogether define the LWR. These metrics are the total root-mean-square (r.m.s.) roughness $\left(\sigma_{\mathrm{LWR}}\right)$, the correlation length $(\xi)$, and the roughness exponent $(\alpha)$. With these metrics, it is possible to quantify the roughness contribution introduced at each spatial frequency.
This methodology brings advantages from the metrology point of view in terms of device reliability monitoring and control. For instance, critical dimension (CD) variations and low-frequency LWR are known to affect intratransistor performance, while high-frequency LWR is known to affect intertransistor performance. Furthermore, device performance has been found not to be affected by the roughness contributions above a specific technology-node dependent frequency (equal to the reciprocal of the pitch) owing to the spatial averaging effects of the device dimension.

The International Technology Roadmap for Semiconductors (ITRS) sets the target requirements for the maximum total LWR that can be tolerated per technology node in an integration process. These LWR targets are summarized in Table 1 for the years 2011 to 2017 . The $\sigma_{\text {LWR }}$ upper limit is set to $1.6 \mathrm{~nm}$ for year $2016^{\text {B }}$ (not taking into account resist smoothing techniques before and during edge transfer). The maximum LWR according to ITRS specification is technology node-dependent and, for the reasons mentioned above, it is calculated in the spatial frequency range from 0 to the cut-off frequency (1/pitch).

In addition to the total $\sigma_{\mathrm{LWR}}$, it is agreed that the correlation length and the roughness exponent also play a role in the device performance, but their effect is not yet fully understood. Nevertheless, extreme ultraviolet (EUV) resist platforms will undoubtedly employ radically different approaches to patterning, which will likely result in different characteristic LWR metrics. It is worth mentioning that the 
Table 1 ITRS target specifications for dimension and LWR of devices.

\begin{tabular}{lcccccccc}
\multicolumn{1}{c}{ Technology } & 2011 & 2012 & 2013 & 2014 & 2015 & 2016 & 2017 \\
\hline DRAM Half pitch $(\mathrm{nm})$ & 36 & 32 & 28 & 25 & 23 & 20 & 18 \\
Flash & Half pitch $(\mathrm{nm})$ & 22 & 20 & 18 & 17 & 15 & 14.2 & 13 \\
Logic & Half pitch $(\mathrm{nm})$ & 38 & 32 & 27 & 24 & 21 & 19 & 17 \\
& LWR $(\mathrm{nm})$ & 2.8 & 2.5 & 2.2 & 2 & 1.8 & 1.6 & 1.4 \\
\hline \hline
\end{tabular}

roughness transfer from the photoresist during plasma etching affects the spatial components of LWR in different ways, ${ }^{\text {Q }}$ which is of technological interest for process integration. The origin of roughness lies in several physical causes that are known. However, it is expected that the different chemical properties of resists affect the roughness metrics in different ways. In the scope of this work, we aim at comparing across platforms of chemically amplified resist (CAR) and inorganic formulations for EUV lithography. The EUV photoresists chosen for this study are state-of-the-art materials specifically designed for high patterning performance; these formulations (or variants based on these formulations) are expected to be used in the EUV lithography process. This work provides a comparative study of the LWR and performance of different photoresists in the light of the roughness metrics, and an interpretation is proposed.

\section{Experimental}

\subsection{Sample Preparation and Description}

Photoresist materials were patterned by EUV interference lithography (EUV-IL) at the XIL beamline of the Swiss Light Source (SLS) using diffraction transmission gratings as described elsewhere. 9 Densely packed line/space (L/S) patterns with pitches ranging from 100 to $36 \mathrm{~nm}$ have been investigated here. Each photoresist was processed according to the manufacturers' specifications: coating conditions, thermal treatments, development, and postprocessing. The list of

Table 2 Description of samples for comparative LWR study.

\begin{tabular}{lccccc}
\hline Resist & Manufacturer & Type & C.A. & $\begin{array}{c}\text { Thickness } \\
(\mathrm{nm})\end{array}$ & Development \\
\hline PMMA & $\begin{array}{c}\text { Sigma } \\
\text { Aldrich }\end{array}$ & Organic & $\mathrm{N}$ & 40 & MIBK:IPA \\
HSQ & Dow Corning & Inorganic & $\mathrm{N}$ & 35 & $\mathrm{NaBOH}$ \\
CAR 1 & Undisclosed & Organic & $\mathrm{Y}$ & 35 & Undisclosed \\
CAR 2 & Undisclosed & Organic & $\mathrm{Y}$ & 35 & Undisclosed \\
CAR 3 & Undisclosed & Organic & $\mathrm{Y}$ & 25 & Undisclosed \\
YABA & Inpria Corp. & Metal-oxide & $\mathrm{N}$ & 32 & Undisclosed \\
YF & Inpria Corp. & Metal-oxide & $\mathrm{N}$ & 25 & Undisclosed \\
\hline \hline
\end{tabular}

samples is shown in Table $\mathrm{Q}$. The polymethyl methacrylate (PMMA, $950 \mathrm{k}$ molecular weight, $1 \%$ in chlorobenzene) and hydrogen silsesquioxane (HSQ, FOX16 diluted in methyl isobutyl ketone 1:6) were chosen as reference systems because of their very well-known properties. Two different alkaline solutions [tetramethylammonium hydroxide (TMAH) and sodium boron hydroxide $(\mathrm{NaBOH})]$ were used to develop HSQ. We tested three state-of-the-art CARs specifically designed for EUV lithography based on organic polymer backbones from undisclosed manufacturers. Finally, we tested two different formulations (code-named YABA and YF) of photocondensed, negative tone, and molecular oxide resists that are nonchemically amplified and synthesized by Inpria Corp. All photoresists were patterned using the same transmission mask and under the same illumination conditions.

\subsection{Analytical Metrology}

The patterned resists were inspected using a scanning electron microscope (SEM) Zeiss Supra VP55, using the following settings: EHT $1 \mathrm{keV}$; beam current $21 \mathrm{pA}$; frames per picture: 1; frame integration: none; and pixel aspect ratio 1:1. The obtained top-down SEM images were then analyzed using SuMMIT software suite to extract the LWR r.m.s. value, correlation length, and roughness exponent. These metrics were calculated using both the PSD and the HHCF descriptors. All results shown in this work are based on the HHCF descriptor because it provided the most consistent output when analyzing noisy images.

The uncertainty in the measurement of these metrics is provided by the algorithm of image analysis. The three roughness metrics were extracted by analysis of SEM images of constant size and different pitch, thus resulting in a data set between 14 and 25 lines per sample. The average of this data set provided a remarkably low uncertainty. For instance, the estimated uncertainties of CD and r.m.s. LWR were consistently below $0.07 \mathrm{~nm}$ for all samples here investigated. The uncertainty of r.m.s. LWR is therefore not indicated in the following plots.

For the calculation of r.m.s. value according to ITRS, a cut-off frequency equal to the reciprocal of the pitch was used. The measurement and analysis of the parameters were performed using the same tool and SEM conditions, to ensure a consistent quantitative comparison of the results.

\section{Results and Discussion}

The PSD of the materials described above was calculated from the SEM images for the entire data set of pitches and doses. For the sake of comparison, Fig. [1] shows the calculated averaged LWR PSD as a function of the spatial frequency for selected data from all samples at the dose-to-size and pitch $44 \mathrm{~nm}$. It can be noticed that all the extracted PSD shows a relatively modest noise, owing to the averaging of a conspicuous set of lines. Moreover, it is possible to notice clearly distinct trends, peculiar to each sample, beyond the uncertainty of the measurement. These differences are particularly clear in the frequency dependence and allow us to confidently proceed in the analysis of the three roughness metrics in detail in the following sections and to draw conclusions on the different resist platforms. 


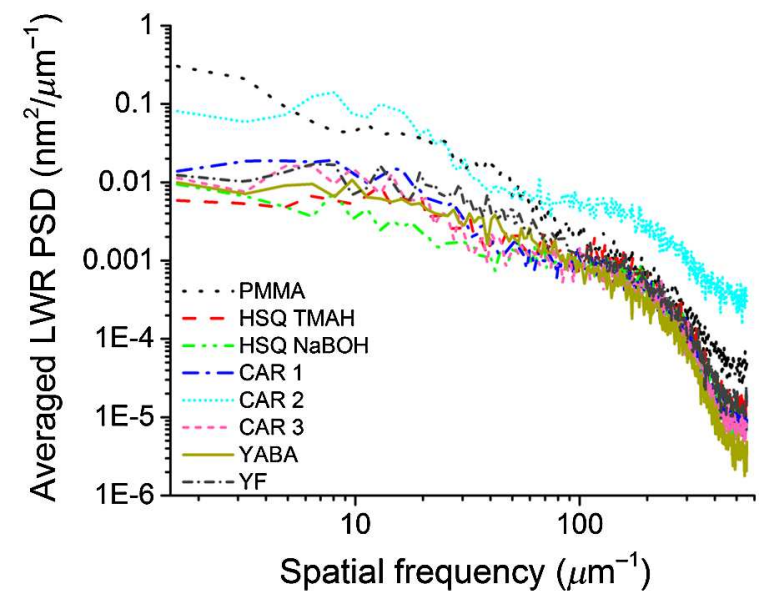

Fig. 1 Averaged LWR PSD of selected samples at the dose-to-size and pitch $44 \mathrm{~nm}$. The differences in the raw data shown here are specific to each resist platform.

\subsection{Total Root-Mean-Square Line Width Roughness}

For comparison purposes, here we consider the total r.m.s. roughness of lines patterned at dose-to-size (also known as Best Energy or $E_{\text {size }}$ ). As a matter of fact, L/S patterns produce the lowest total roughness when the exposure dose used is equal to the dose-to-size because the slope of the quasibeam profile is the highest. The total LWR for each resist at every pitch tested is shown in Fig. \&. From the plot, it is clear that the roughness is not significantly affected by the pitch size. This is due to the fact that the produced aerial image by interference lithography has a constant normalized image log slope, independent of the pitch. As a result, EUV-IL is a powerful technique to compare the roughness of $\mathrm{L} / \mathrm{S}$ images at different pitches. It can be noticed from Fig. \& that the r.m.s. LWR mostly depends on the specific feature of the resist. In this regard, remarkable differences can be noted. HSQ was the material that yielded the lowest total roughness followed by the YABA and YF materials: the small molecular building block of these three materials contributes to the achievement of such a low LWR. Performance of the three CAR resists varied greatly and depended on the acid diffusion length and polymer size. In the case of PMMA, the high roughness can be ascribed to the large molecular size of the polymer, which was in this case a molecular weight of $950 \mathrm{k}$.

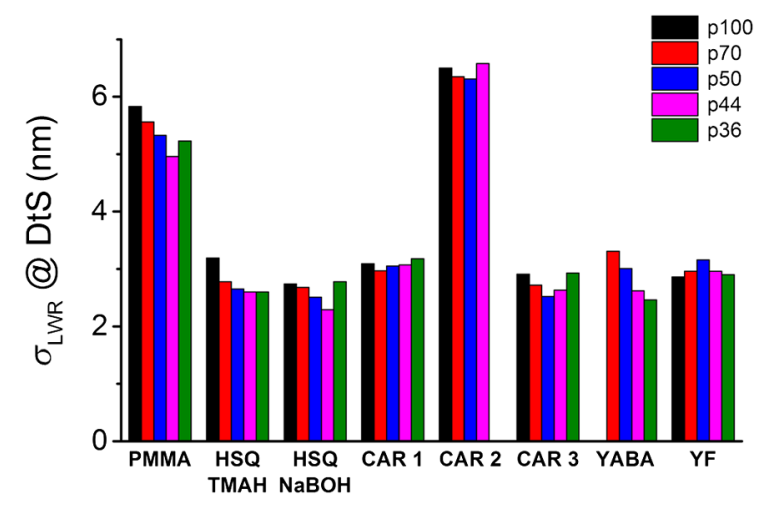

Fig. 2 Total r.m.s. LWR measured for each resist at the dose-to-size for pitches 100 to $36 \mathrm{~nm}$.

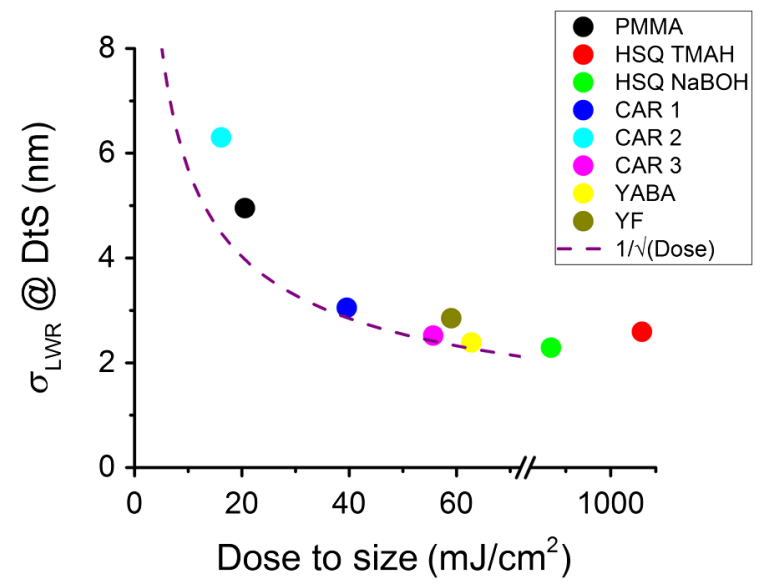

Fig. 3 Total r.m.s. LWR (averaged over all pitches) as a function of the dose-to-size for each resist (symbols). The RLS trade-off is shown in dashed line as a guide to the eye.

The total LWR of these resist platforms is shown in relation to the specific dose to size in Fig. 3. It is widely known that the RLS trade-off relationship postulates that the three parameters of resolution, roughness, and sensitivity of a photoresist cannot be improved simultaneously. Specifically, the limitation to the reduction of LWR is the sensitivity; at low doses, the photon shot noise statistics (where the spatial fluctuations of photons on the photoresist) cause an increase in LWR. This trade-off is shown in Fig. 3 by the well-known relationship $1 / \sqrt{ }$ Dose, in dashed plot as a guide to the eye. Remarkably, these resists loosely follow this trend despite the differences in the formulations. However, because they have different optical absorption, we cannot conclude that the LWR is in the photon statistics-limited regime and the photon shot noise is not the major source of roughness. Indeed the LWR is also affected by the roughness of the aerial image and by mechanical vibrations during the exposure. The uncertainty in the data is smaller than the dot size for all resists investigated and it is not shown in the plot.

In the framework of the ITRS requirements, let us now consider the $\sigma_{\mathrm{LWR}}$ measured up to a cut-off spatial frequency equal to the reciprocal of the pitch. The resulting $\sigma_{\mathrm{LWR}}$ is shown in Fig. 田. The ITRS-relevant $\sigma_{\mathrm{LWR}}$ is clearly lower

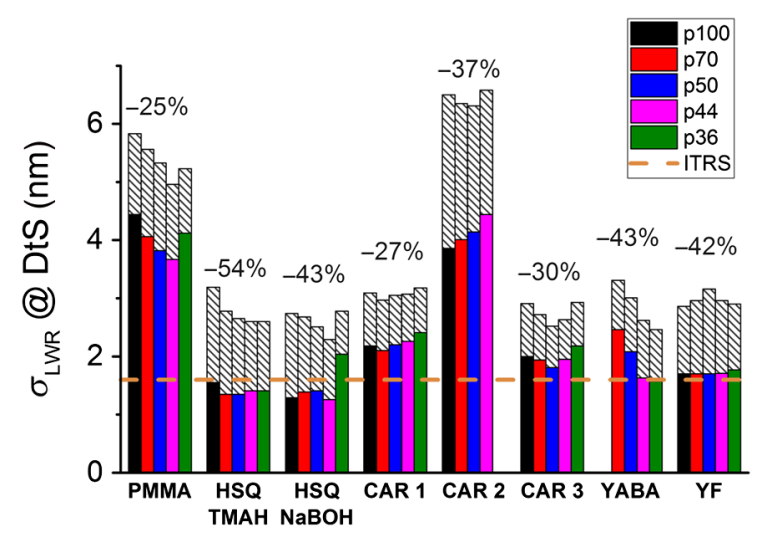

Fig. $4 \sigma_{\mathrm{LWR}}$ calculated according to the ITRS specifications and at the dose-to-size for each resist per pitch from 100 to $36 \mathrm{~nm}$. The dashed orange line indicates the ITRS requirement for year 2016. The numbers indicate the percent difference between the full LWR and the ITRS-LWR (averaged throughout pitches). 
than the full $\sigma_{\mathrm{LWR}}$ because it is measured in a smaller frequency range. Nonetheless, it is remarkably smaller in the case of nonpolymer resists $(-40 \%$ to $-54 \%)$ than it is for all others resists $(-25 \%$ to $-37 \%)$. This observation leads us to conclude that the chemical composition affects the frequency components differently and should be taken into account for the device performance. Finally, we note that the ITRS specification is at present quite challenging to be met for all EUV resists, unless additional smoothing techniques are employed.

\subsection{Correlation Length $\xi$}

The $\xi$ metrics represents the boundary between the low-frequency regime (plateau of the PSD) and the high-frequency regime (self-affine geometry).

The correlation length of each sample was extracted from the HHCF of each line edge, calculated in the domain of spatial frequencies. The data analysis algorithm automatically calculated the spatial frequency $f$ at which the regime of the HHCF changes from fractal $(f>1 / \xi)$ to white noise $(f<1 / \xi)$. Since the $\xi$ is extracted from a data set of at least 14 lines, its standard deviation was always below $1 \mathrm{~nm}$ for all samples and it is not shown in Fig. 5. It is agreed that the size of the molecule, the acid diffusion, and the development all affect the correlation length. The statistical distribution of measured $\xi$ values of each resist is shown in Fig. 5 .

The general trend seems to indicate that polymer-based resists (PMMA, CAR 1, CAR 2, and CAR 3) have larger $\xi$ than nonpolymers (HSQ, YABA, and YF). In fact, the molecular size of the former resists is defined by the radius of gyration of their backbone polymer. In PMMA of molecular weight $950 \mathrm{k}$, the radius of gyration can be as large as $30 \mathrm{~nm}$, while for these CAR resists it is estimated $<10 \mathrm{~nm}$. Moreover, acid diffusion also plays a role in CARs and adds to the $\xi$, which explains why their correlation length ( $\approx 10$ to $12.5 \mathrm{~nm}$ ) is comparable to that of the nonchemically amplified materials, but larger than for the polymer PMMA.

\subsection{Roughness Exponent $\alpha$}

The $\alpha$ was extracted by the algorithm as the slope of the linear fit to the HHCF in the fractal regime, i.e., at spatial frequencies $f$ greater than $1 / \xi$. Because $\alpha$ is extracted

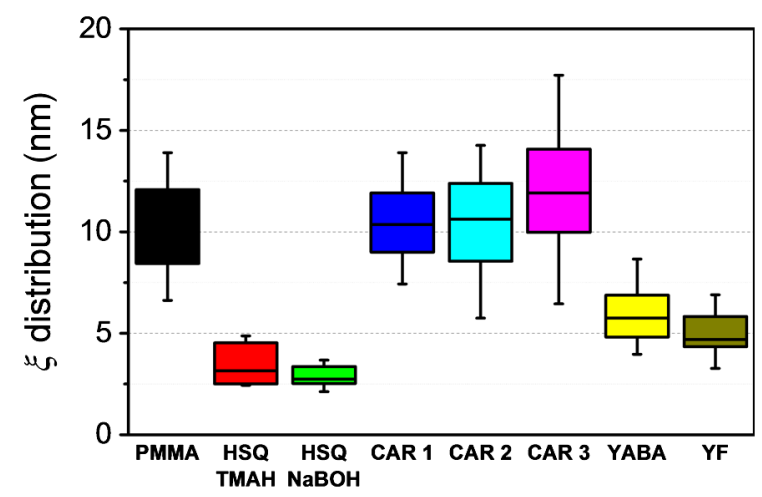

Fig. 5 Statistical distribution of the correlation length for all samples. Boxes indicate the median and top/lower 25th percentile, while caps indicate the top/lower fifth percentile.

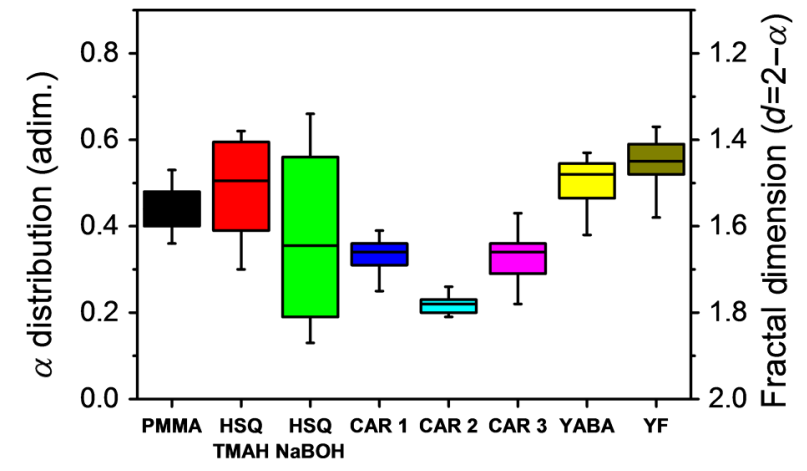

Fig. 6 Distribution of the roughness exponent $\alpha$ (and corresponding fractal dimension $d=2-\alpha$ ) for all resists investigated in this work. Boxes indicate the median and top/lower 25th percentile, while caps indicate the top/lower fifth percentile.

from a data set of at least 14 lines, its standard deviation was always smaller than 0.03 for all samples and it is not shown in Fig. 6. The meaning of $\alpha$ in the fractal regime is usually discussed in the framework of fractal geometry. Indeed, several studies found that submicrometric surfaces and structures exhibit self-affine morphologies typical of fractals $\$$ For line edges, the fractal dimension $d$ is then defined in relationship to the roughness exponent $\alpha$ according to the relation $d=2-\alpha$. In self-affined geometries, the fractal dimension $d$ is always larger than the topological dimension ( 1 for edges) and even larger when the edge complexity is higher.

The statistical distribution of roughness exponent $\alpha$ (and corresponding fractal dimension $d$ ) of all samples is shown in Fig. 6.

In the fractal regime, all CARs yielded the line edges with the most complex contour, which we attribute to the effect of acid diffusion. In addition, a smaller $\alpha$ might indicate that the dissolution process is more homogeneous in CAR resists than it is in PMMA (which is a larger polymer and not as uniformly dissolved along the resist sidewalls). $\square$ The HSQ yielded different roughness depending on the development process, which seems to affect $\alpha$ much more than the other metrics. In this regard, it is proposed that the slower developer $(\mathrm{NaBOH})$ might form higher complex contours than TMAH does. Finally, the metal oxides materials (YABA and YF) formed lines with the lowest complexity among all examined here.

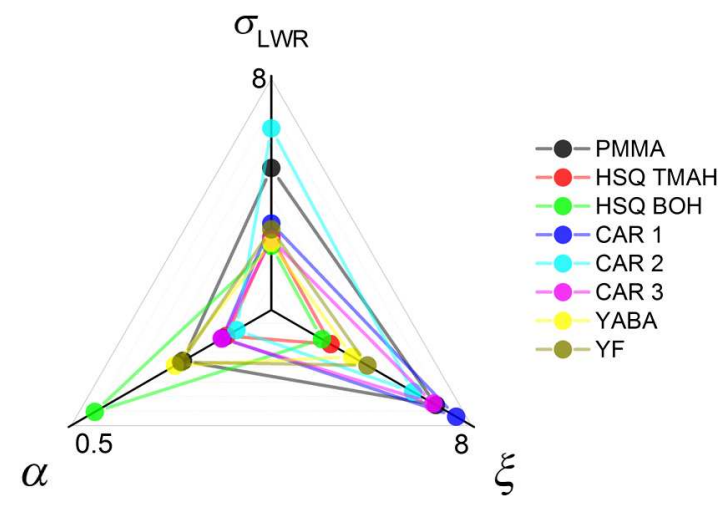

Fig. 7 Summary of the results for the three metrics of resists investigated in this work. 


\subsection{Summary of Results}

Different resist platforms showed remarkable differences in their roughness metrics due to the diverse chemical properties of each formulation. The summary of the results is shown in Fig. 7.

\section{Conclusions}

We have measured the LWR metrics of different resist platforms using a unified metrological approach. The LWR metrics change considerably depending on the lithographic process and according to the specific physicochemical properties, molecular size, and development of each material.

It was found that the correlation length is mostly affected by the combination of polymer size and acid diffusion. The roughness exponent is mostly affected by the development process and by the acid diffusion. These observations let us conclude that remarkable differences are found in the roughness features of CAR and inorganic resists.

From the point of view of device manufacturing and reliability, the effect of $\xi$ and $\alpha$ on the device performance is of great technological interest. In this regard, theoretical calculations indicate that lower $\xi$ and $\alpha$ minimize the detrimental effect on performance of devices. ${ }^{12}$ Further studies are required to experimentally assess these effects on manufactured devices.

\section{Acknowledgments}

The authors are grateful to Jason Stowers (Inpria Corp.) for fruitful discussions and to Michaela Vockenhuber (PSI) for sample analysis and fruitful discussions. Part of this work was carried out at SLS. Inpria Corp. is acknowledged for financial support.

\section{References}

1. G. Leung et al., "Device- and circuit-level variability caused by line edge roughness for sub-32-nm FinFET technologies," IEEE Trans. Electron Devices 59(8), 2057-2063 (2012).

2. V. Constantoudis et al., "Quantification of line-edge roughness of photoresists. II. Scaling and fractal analysis and the best roughness descriptors," J. Vac. Sci. Technol. B 21(3), 1019-1026 (2003).

3. "International technology roadmap for semiconductors," 2013, www. itrs2.net/2013-itrs.html (March 2014).

4. S. Levi et al., "Edge roughness characterization of advanced patterning processes using power spectral density analysis (PSD)," Proc. SPIE 9782, 97820I (2016).

5. G. F. Lorusso et al., "Line-edge roughness accuracy analysis during pattern transfer in self-aligned quadruple patterning process," Proc. SPIE 9778, 97780V (2016).

6. E. Buitrago et al., " $\mathrm{SnO}_{\mathrm{x}}$ high-efficiency EUV interference lithography, gratings towards the ultimate resolution in photolithography," Microelectron. Eng. 155, 44-49 (2016).

7. M. Yoshizawa and S. Moriya, "Edge roughness evaluation method for quantifying at-size beam blur in electron-beam lithography," Proc. SPIE 3997, 301-308 (2000).

8. M. Kamijo et al., "Mean-square radius of gyration of isotactic oligo- and Poly(methyl methacrylate)s in dilute solution," Macromolecules 27(20), 5697-5703 (1994).

9. A.-L. Barabási and H. E. Stanley, Fractal Concepts in Surface Growth, 1st ed., Cambridge University Press, Cambridge, United Kingdom (1995).

10. J. M. Li et al., Image-Based Fractal Description of Microstructures, Springer Science+Business Media, New York (2003).

11. V. Constantoudis, G. P. Patsis, and E. Gogolides, "Evolution of resist roughness during development: stochastic simulation and dynamic scaling analysis," J. Micro/Nanolithogr. MEMS MOEMS 9(4), 041207 (2010).

12. G. P. Patsis, V. Constantoudis, and E. Gogolides, "Integrated simulation of line-edge roughness (LER) effects on sub-65nm transistor operation: from lithography simulation, to LER metrology, to device operation," Proc. SPIE 6151, 61513J (2006).

Biographies for the authors are not available. 